\title{
Numerical algorithm for solving of nonlinear problems of structural mechanics based on the continuation method in combination with the dynamic relaxation method
}

\author{
Sergey Trushin ${ }^{1, *}$ \\ ${ }^{1}$ NRU MGSU, Department of Structural and Theoretical Mechanics, Yaroslavskoe sh. 26, 129337 \\ Moscow, Russia
}

\begin{abstract}
A numerical algorithm of strength and stability analysis of nonlinear deformable bar systems and thin-walled spatial structures is proposed. A numerical technique is based on the continuation method and the dynamic relaxation method. When using the method of dynamic relaxation the state of static equilibrium of structures is defined after the damped oscillations by integrating over leading parameter. The solution of the initial equation system describing the motion of the mechanical system is reduced to the solution of Cauchy problem for systems of ordinary differential equations. At an each step in the leading parameter the vector of nodal displacements and the time parameter are defined. Several examples of numerical analysis for bar, shell and plate are given.
\end{abstract}

\section{Introduction}

In the study of stability of bar systems and thin-walled structures in a nonlinear formulation there is a need to determine the maximum and bifurcation points on equilibrium curves and study the behavior of structures in an overcritical stage. There is a very effective class of methods for creating the equilibrium curves and studying of stability forms of equilibrium of structures. The main idea of these methods is to find a sequence of solutions $\mathbf{u}_{m}=\mathbf{u}\left(p_{m}\right)$ $(m=0,1,2, \ldots, M)$ of the system of nonlinear equations $\mathbf{F}(\mathbf{u}, p)=0$ on the basis of available initial solution $\mathbf{u}_{0}=\mathbf{u}\left(p_{0}\right)$ when the leading parameter $p$ is changed from $p_{0}$ to $p_{M}$. Various variants of this approach were developed by the researchers [1-5] and several other authors. The continuation method in stability problems of isotropic shells were used in the works [6, 7].

Analysis of the solutions of nonlinear problems of the theory of plates and shells has shown that different schemes based on the Newton-Raphson or Runge-Kutta procedures are given the best results. They use an arc length of the equilibrium curve as the leading parameter and include an auxiliary equation for the iteration on the sphere. Using an arc length as a parameter of the continuation provides an unified procedure of passing through regular, limit and bifurcation points. There is no need to change the leading parameter when

*Corresponding author: trushin2006@yandex.ru 
passing a limit point and the very notion of a limit point in this formulation is meaningless.

However, the approach based on the implicit procedures of the Newton-Raphson method has, like any other, certain disadvantages. In particular, when such numerical procedures are used the stiffness matrix becomes singular near critical points.

\section{Solution method}

When solving static problems with strongly nonlinearity an efficient numerical algorithm based on a combination of the continuation method and the method of dynamic relaxation can be built. The main idea is that the static equilibrium of structures is defined after the damped oscillations with help of integration by time [8-10]. Thus, for solving problems of static stability the equations of motion can be used.

The equations describing the motion of the mechanical system have the form:

$$
\mathbf{M x}+\mathbf{C x}+\mathbf{K x}=\mathbf{P}(t)
$$

where $\mathbf{x}$ is the displacement vector; $\mathbf{M}$ is the mass matrix; $\mathbf{C}$ is the damping matrix; $\mathbf{K}$ is the stiffness matrix of the system; $\mathbf{P}(t)$ is the vector of nodal loads.

We introduce the notation $\mathbf{z}_{1}=\mathbf{x}$ and $\mathbf{z}_{2}=\mathbf{z}_{1}=\mathbf{v}$ where $\mathbf{v}$ is the velocity vector. Transforming the equation (1) as shown below:

$$
\left\{\begin{array}{l}
\mathbf{z}_{1}=\mathbf{z}_{2} \\
\mathbf{z}_{2}=-\mathbf{M}^{-1}\left(\mathbf{K z}_{1}+\mathbf{C} \mathbf{z}_{2}-\mathbf{P}(t)\right)
\end{array}\right.
$$

or

$$
\mathbf{z}=\mathbf{f}(\mathbf{z}, t)
$$

where

$$
\grave{\mathbf{z}}=\left(\grave{\mathbf{z}}_{1} \mathbf{z}_{2}\right)^{T}, \mathbf{z}=\left(\mathbf{z}_{1} \mathbf{z}_{2}\right)^{T}, \mathbf{f}=\left(\begin{array}{c}
\mathbf{z}_{2} \\
-\mathbf{M}^{-1}\left(\mathbf{K}_{1}+\mathbf{C} \mathbf{z}_{2}-\mathbf{P}(t)\right)
\end{array}\right) .
$$

We transform the vector $\mathbf{f}$ to the form:

$$
\mathbf{f}=\left(\begin{array}{c}
\mathbf{z}_{2} \\
-\mathbf{M}^{-1}\left(\mathbf{C} \mathbf{z}_{2}-\mathbf{R}\right)
\end{array}\right)
$$

where $\mathbf{R}=\mathbf{K} \mathbf{z}_{1}-\mathbf{P}(t)$ represents the residual of the solution of static problem.

Assuming that the nodal displacements $\mathbf{x}$ and time $t$ depend on some parameter $s$, we introduce an additional equation for the iterations on the cylindrical surface in the system (3):

$$
\left\{\begin{array}{l}
\frac{d \mathbf{z}}{d t}=\mathbf{f}(\mathbf{z}, t) \\
\left(\frac{d \mathbf{z}}{d s}, \frac{d \mathbf{z}}{d s}\right)=1
\end{array}\right.
$$


Multiplying the first equation of system (6) by $\frac{d t}{d s}$, we get:

$$
\frac{d \mathbf{z}}{d s}=\mathbf{f}(\mathbf{z}, t) \frac{d t}{d s}
$$

Substituting (7) into the second equation of system (6) we have:

$$
\left(\frac{d t}{d s}\right)^{2}(\mathbf{f}, \mathbf{f})=1
$$

or

$$
\frac{d t}{d s}=\frac{1}{\sqrt{(\mathbf{f}, \mathbf{f})}}
$$

Substituting (9) into (7) we get finally an ordinary differentiation system with the initial conditions:

$$
\left\{\begin{array}{l}
\frac{d \mathbf{x}}{d s}=\frac{\mathbf{v}}{\sqrt{(\mathbf{f}, \mathbf{f})}} \\
\frac{d \mathbf{v}}{d s}=-\frac{1}{\sqrt{(\mathbf{f}, \mathbf{f})}} \mathbf{M}^{-1}(\mathbf{K} \mathbf{x}+\mathbf{C v}-\mathbf{P}(t)) \\
\frac{d t}{d s}=\frac{1}{\sqrt{(\mathbf{f}, \mathbf{f})}} \\
\mathbf{x}(0)=\mathbf{x}_{0} \\
\mathbf{v}(0)=\mathbf{v}_{0} \\
t(0)=t_{0}
\end{array}\right.
$$

For the solution of the Cauchy problem (10) Runge-Kutta or Euler methods can be used. Scheme of Euler method has the form:

$$
\left\{\begin{array}{l}
\mathbf{v}_{k+1}=\mathbf{v}_{k}-\frac{\Delta s}{\sqrt{\left(\mathbf{f}_{k}, \mathbf{f}_{k}\right)}} \mathbf{M}^{-1}\left(\mathbf{R}_{k}+\mathbf{C} \mathbf{v}_{k}\right) \\
\mathbf{x}_{k+1}=\mathbf{x}_{k}+\frac{\Delta s}{\sqrt{\left(\mathbf{f}_{k}, \mathbf{f}_{k}\right)}} \mathbf{v}_{k+1} \\
t_{k+1}=t_{k}+\frac{\Delta s}{\sqrt{\left(\mathbf{f}_{k}, \mathbf{f}_{k}\right)}} \\
\mathbf{v}(0)=\mathbf{v}_{0} \\
\mathbf{x}(0)=\mathbf{x}_{0} \\
t(0)=t_{0}
\end{array}\right.
$$

The solution of scheme (11) gives the opportunity to find the components of displacement vector $\mathbf{x}$ and time parameter $t$ in each step of leading parameter $s$.

If $\mathbf{C}=0$, then 


$$
\mathbf{f}=\left(\begin{array}{c}
\mathbf{z}_{2} \\
-\mathbf{M}^{-1} \mathbf{R}
\end{array}\right)
$$

and the scheme of Euler method will be presented in the form:

$$
\left\{\begin{array}{l}
\mathbf{v}_{k+1}=\mathbf{v}_{k}-\frac{\Delta s}{\sqrt{\left(\mathbf{f}_{k}, \mathbf{f}_{k}\right)}} \mathbf{M}^{-1} \mathbf{R}_{k} \\
\mathbf{x}_{k+1}=\mathbf{x}_{k}+\frac{\Delta s}{\sqrt{\left(\mathbf{f}_{k}, \mathbf{f}_{k}\right)}} \mathbf{v}_{k+1} \\
t_{k+1}=t_{k}+\frac{\Delta s}{\sqrt{\left(\mathbf{f}_{k}, \mathbf{f}_{k}\right)}} \\
\mathbf{v}(0)=\mathbf{v}_{0} \\
\mathbf{x}(0)=\mathbf{x}_{0} \\
t(0)=t_{0}
\end{array}\right.
$$

\section{Numerical analysis}

\subsection{Straight bar}

The straight bar with a constant cross section under the action of longitudinal forces is considered. Initial data: $M=1, C=0, K=1, P=1, \Delta s=0,01$. The analysis is performed with help of the procedure (13).

The displacement $x$, velocity $v$, kinetic energy $T=M v^{2} / 2$ and residual $R=K x-P$ are shown in Fig. 1. The solution is obtained at 157 steps of leading parameter $s$.

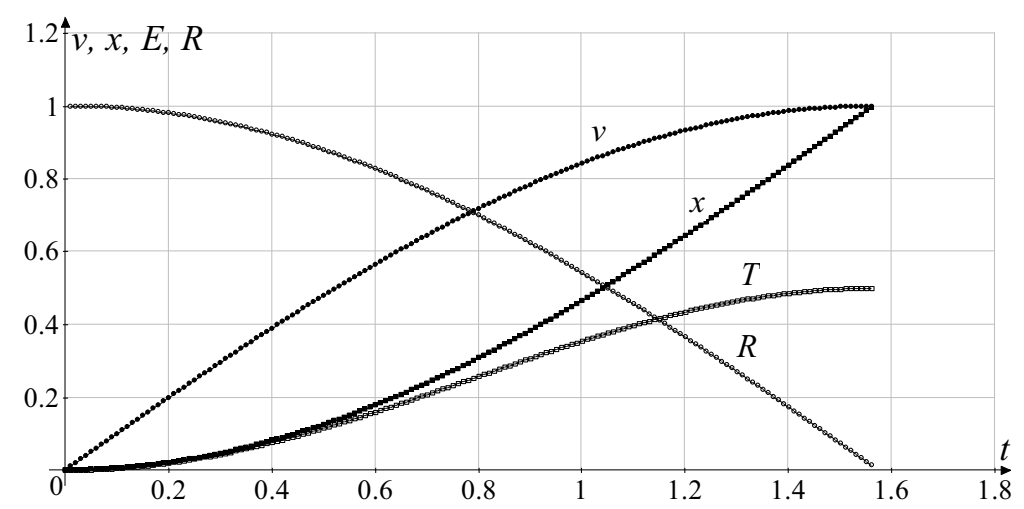

Fig. 1. The results of solution problem of straight bar deformation.

\subsection{Shell and plate}

Deformation of a flexible spherical shell on a rectangular plan under an action of uniformly distributed transverse load intensive $q$ is considered (Fig. 2).

Boundary conditions are hinged support along the contour. Shallow shell sizes on the 
plane $l_{1}$ and $l_{2}$, the radius of curvature $R$, thickness $h$, elastic modulus of the material $E$ are given.

The solution of nonlinear differential equations in the mixed form made by the BubnovGalerkin method is used [11]. In accordance with a given approximating function the maximum deflection $f$ will be in the center of the shell.

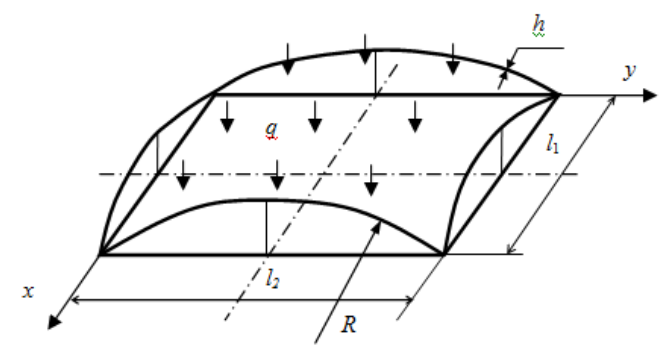

Fig. 2. Shallow shell.

For a shell on a square plan if $l_{1}=l_{2}$ the dependence between the nondimensional load parameter $P$ and the nondimensional deflection at the centre $x$ when Poisson's ratio $v=0,3$ is:

$$
\left(8.77 x^{2}-2.46 k x+0.154 k^{2}+22\right) x=P,
$$

where

$$
k=\frac{2 l_{1}^{2}}{R h}, P=\frac{q}{E}\left(\frac{l_{1}}{h}\right)^{4}, x=\frac{f}{h} .
$$

The solution is performed using the procedure (11) with increment $\Delta P$. The data for the analysis of shell is follows: $M=10^{4}, C=10, \Delta P=50, k=36, \Delta s=0,01$.

The equilibrium curve of the flexible shell is shown in Fig. 3. On the fourth step "snapthrough" of shell takes place and there is a transition into a postbuckling equilibrium state.

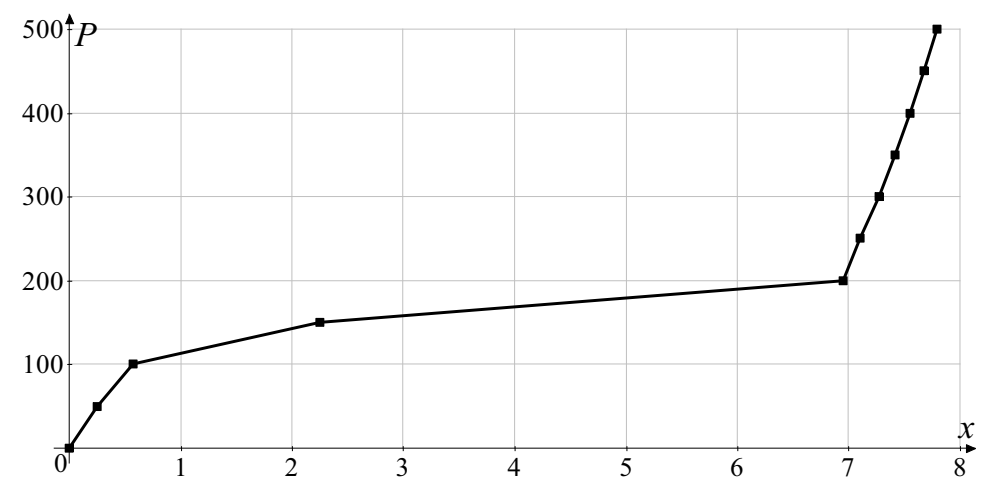

Fig. 3. The equilibrium curve of shallow shell

From the expression (14) as a particular case the solution for flexible plates should be. The data for the analysis of plate is follows: $M=10^{4}, C=10, \Delta P=10, k=0, \Delta s=0,01$. The 
equilibrium curve of the flexible plate is shown in Fig. 4.

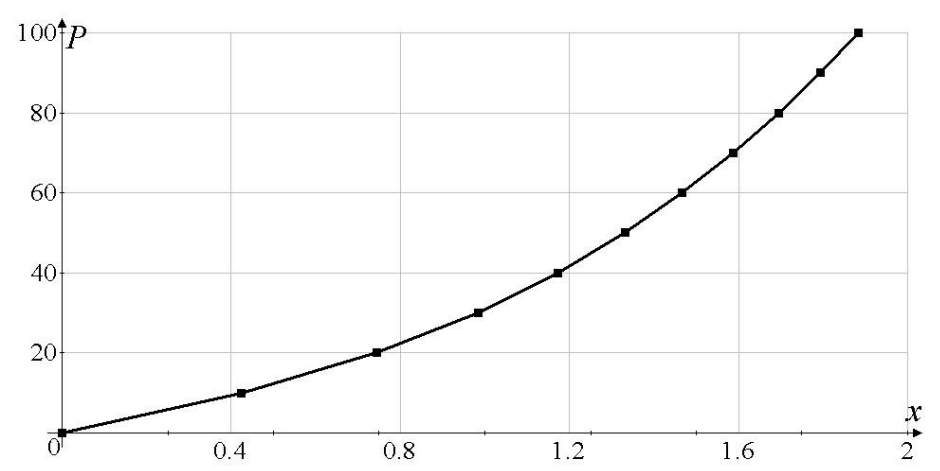

Fig. 4. The equilibrium curve of plate.

\section{Conclusion}

The continuation method is designed for building of equilibrium curves, determination of limit and bifurcation loads and evaluation of stability of equilibrium in nonlinear analysis of bar systems, plates and shells. One of the most effective numerical methods for solving these types of problems with help of the continuation method is a procedure in which at each step the solution is corrected by Newton-Raphson method. However, using such implicit algorithms may cause some difficulties in numerical implementation of the solution as the stiffness matrix becomes singular near critical points. So the explicit algorithm of dynamic relaxation method can be successfully applied in the analysis of nonlinear behavior of structures near critical points. It does not use the procedure of a matrix inversion or the solution of simultaneous linear algebraic equations with the matrix $\mathbf{K}$ in the formulation (10).

The fundamental feature of the dynamic relaxation method is that the condition of static equilibrium of the structure is determined after its damped oscillations with help of quasistatic integration by time or by another leading parameter. But with the rejection of the use of matrix operations connected the main disadvantage of the dynamic relaxation method, consisting in a relatively low speed of convergence of the solution.

\section{References}

1. M.A. Crisfield, Comput. Struct. 13, 55 (1981)

2. E. Ricks, J. Appl. Mech. 39, 1060 (1972)

3. G.A. Wempner, Int. J. Sol. Struct. 7, 1581 (1971)

4. J.L. Batoz, G. Dhatt, Int. J. Numer. Meth. Eng. 14, 1262 (1979)

5. V.V. Petrov, Nonlinear incremental structural mechanics (Infra-Inzheneriya, Moscow, 2014)

6. I.E. Mileikowskii, S.I. Trushin, Analysis of Thin-Walled Structures (A.A. Balkema Publishers, Rotterdam, 1994)

7. S.I. Trushin, Structural mechanics: finite element method (INFRA-M, Moscow, 2016)

8. A.S. Day, The Engineer 219, 218 (1965)

9. G. Ramesh, C.S. Krishnamoorthy, Int. J. Numer. Meth. Eng. 36, 1339 (1993)

10. K.S. Lee, S.E. Han, T. Park, Comput. Struct. 89, 216 (2011)

11. A.S. Vol'mir, Stability of deformable systems (Scince, Moscow, 1967) 